\title{
SUPERGRAVITY AND THE QUEST FOR A UNIFIED THEORY
}

\author{
Sergio Ferrara \\ Theoretical Physics Division, \\ CERN, Geneva, Switzerland
}

\begin{abstract}
A recollection of some theoretical developments that preceded and followed the first formulation of supergravity theory is presented. Special emphasis is placed on the impact of supergravity on the search for a unified theory of fundamental interactions.
\end{abstract}

Dirac Lecture delivered at

the International Centre for Theoretical Physics, Trieste, 19 April 1994 

It is a great honour and pleasure to be invited to give this Dirac Lecture on the occasion of the 1994 Spring School on String, Gauge Theory and Quantum Gravity.

In fact, this School is a continuation of a very successful series initiated by Prof. A. Salam in 1981. Together with J.G. Taylor and P. van Nieuwenhuizen I had the privilege of organizing the first two in the spring of 1981 and the fall of 1982 [1].

At that time, supergravity was in the mainstream of research, namely

1) Quantum properties of extended supergravities and their geometric structure,

2) Kaluza-Klein supergravity,

3) Models for particle physics phenomenology.

These topics were widely covered during the first two schools and workshops.

Before going on to discuss supergravity and its subsequent development, let me briefly touch upon the steps taken in the two preceding years, when supersymmetry in four dimensions was introduced.

Although the latter, with its algebraic structure, was first mentioned in 1971 by Gol'fand and Likhtman [2] and in early 1973 by Volkov and Akulov [3] (to explain the masslessness of the neutrino as a Goldstone fermion), it was really brought to the attention of theoretical particle physicists in the second half of 1973, by Wess and Zumino [4]; they had been inspired by a similar structure, found by Gervais and Sakita (1971) [5], already present in two dimensions, in the dual-spinor models constructed in 1971 by Neveu and Schwarz [6] and by Ramond [7]. The relevance of supersymmetry for quantum field theory, especially in view of its remarkable ultraviolet properties and its marriage with Yang-Mills gauge invariance, was soon established in early 1974.

It is nevertheless curious that it was only, at the time, rather isolated groups that delved into the subject, mainly in Europe: at CERN, the ICTP (Trieste), Karlsruhe, the ENS-Paris, Imperial College-London, Turin Univ., and essentially two in the United States: Caltech and Stony Brook. The same applies to supergravity and its ramifications in the early years, after its foundation in 1976.

Soon after the very first paper of Wess and Zumino [8], a remarkable sequence of events occurred during 1974:

- The superspace formulation of supersymmetric field theories (Salam, Strathdee [9]; Wess, Zumino, Ferrara [10]).

- The discovery of non-renormalization theorems (Wess, Zumino [11]; Iliopoulos, Zumino [12]; Ferrara, Iliopoulos, Zumino [13]).

- The construction of supersymmetric Yang-Mills theories (Wess, Zumino [14 for the Abelian case; Ferrara, Zumino [15], and Salam, Strathdee [16], for the non-Abelian case). 
- The first construction of a renormalizable field theory model with spontaneously broken supersymmetry (Fayet, Iliopoulos [17]).

- The construction of a multiplet of currents, including the supercurrent and the stress energy tensor (Ferrara, Zumino [18]), which act as a source for the supergravity gauge fields and had an impact also later, in the classification of anomalies and in the covariant construction of superstring Lagrangians.

In the same year, quite independently of supersymmetry, Scherk and Schwarz [19] proposed string theories as fundamental theories for quantum gravity and other gauge forces rather than for hadrons, turning the Regge slope from $\alpha^{\prime} \sim \mathrm{GeV}^{-2}$ to $\alpha^{\prime} \sim 10^{-34} \mathrm{GeV}^{-2}$, the evidence being that any such theory contained a massless spin 2 state with interactions for small momenta as predicted by Einsteins' theory of general relativity.

In the following year, many models with spontaneously broken supersymmetry and gauge symmetry were constructed, mainly by Fayet [20] and O'Raifeartaigh [21], and $N=2$ gauge theories coupled to matter, which were formulated by Fayet [22].

This was a prelude to two importants events, which took place just before and soon after the proposal of supergravity: the discovery of extended superconformal algebras (Ademollo et al., Nov. 1975 [23]) and the finding of evidence for space-time supersymmetry in superstring theory (Gliozzi, Olive, Scherk, GOS, for short, Sept. 1976 and Jan. 1977 [24]). In retrospect, these episodes had a great impact on the subsequent development of string theories in the mid 80's:

The Ademollo et al. paper, just a few months before supergravity was formulated, was inspired by the fact that it was possible, in higher dimensions $(D=4)$, by undoing the superspace coordinate $\theta_{i}$ with a counting index $(i=1, \ldots, N)$, to construct extended supersymmetries; indeed, a remarkable theory with $N=2(D=4)$ supersymmetry then had just been discovered by Fayet (Sep. 1985 [22]). In $D=4$, extended superconformal algebras were accompanied by $\mathrm{U}(N)$ gauge algebras [SU(4) for $N=4$ ]. In $D=2$, superconformal algebras are infinite-dimensional and $N=2$ and $N=4$ turned out to be accompanied by U(1) and $\mathrm{SU}(2)$ Kac-Moody gauge algebras. These algebras, at the time thought of as gauge-fixing of superdiffeomorphisms, were introduced to study new string theories with different critical dimension [25]. In retrospect, this construction had a major impact on the classification of "internal" superconformal field theories, especially $N=2$, as the quantum version of Calabi-Yau manifolds, and on its relation [26] with the existence of space-time supersymmetry in $D<D_{\text {crit }}$.

Meanwhile, in the spring of 1976 [27], supergravity was formulated by Freedman, van Nieuwenhuizen and the author, working at the Ecole Normale and at Stony Brook. Soon after, a simplified version (first-order formulation) was presented by Deser and Zumino [28]. 
While in the second formalism, the spin-3/2 four-fermion interaction has the meaning of a contact term (similar to seagull terms in scalar electrodynamics or non-Abelian gauge theories) required by fermionic gauge invariance, in the first-order formalism it has the meaning of a torsion contribution to the spin connection from "spin-3/2 matter". This discovery also implied that any supersymmetric system coupled to gravity should manifest local supersymmetry.

This observation eventually led some physicists to go deeper in string theory in order to explore whether the "dual spinor model" could accommodate target-space supersymmetry. The GOS paper (Sept. 1976 and Jan. 1977 [24]) gave dramatic evidence for space-time supersymmetry in the superstring theory (called at that time the dual-spinor model) [29] by cutting out the $G$-odd parity states in the N-S sector and comparing its bosonic spectrum with the fermion spectrum of the Ramond sector. In the proof, they used an identity that had been proved by Jacobi in 1829 (Aequatio identica satis abstrusa)! This paper came out following some sequential developments in supergravity, just after its first construction in the spring of 1976, namely the first matter-coupling to Maxwell theory (Ferrara, Scherk, van Nieuwenhuizen, Aug. 1976 [30]), to Yang-Mills theory and chiral multiplets [31] and the first formulation of extended supergravity $[N=2]$ (Ferrara, van Nieuwenhuizen, Sept. 1976 [32]). It is interesting to note that two of the GOS authors ( $\mathrm{G}$ and $\mathrm{S}$ ) also took part in some of the above-mentioned supergravity papers.

The hypothesis of GOS (later proved in great detail by Green and Schwarz [33]) also implied the existence of an $N=4$ Yang-Mills theory, eventually coupled to an $N=4$ extended supergravity. This was implied by a dimensional reduction of the $10 D$ spectrum. The full $N=$ 4 supergravity contained in this reduction was found a year later (Cremmer, Scherk, Ferrara, Dec. 1977 [34]) and it was shown to contain an SU(4) $\times$ SU(1,1) symmetry. Meanwhile, three other important developments were announced at the end of 1976. The construction of $N$ = 3 supergravity (Freedman; Ferrara, Scherk, Zumino, Nov. 1976 [35]) and the discovery of (Abelian and non-Abelian) duality symmetries, generalizing the electromagnetic duality $\mathrm{F} \rightarrow \tilde{\mathrm{F}}$ in $N=2[\mathrm{U}(1)]$ and $N=3[\mathrm{U}(3)]$ supergravity (Dec. $1976[36])$. This duality generalizes to $\mathrm{SO}(6) \times \mathrm{SU}(1,1)$ in pure $N=4$ supergravity and to $\mathrm{SO}(6, n) \times \mathrm{SU}(1,1)$ in $N=4$ supergravity coupled to $n$ matter (Yang-Mills) multiplets.

In retrospect, these symmetries play a crucial role in compactified superstrings, where the manifold

$$
\frac{\mathrm{SO}(6, N)}{\mathrm{SO}(6) \times \mathrm{SO}(N)} \times \frac{\mathrm{SU}(1,1)}{\mathrm{U}(1)}
$$

(modded out by some discrete symmetries) describes the moduli space of toroidally compactified $10 D$ strings, according to the analysis of Narain, Sarmadi, Witten [37]).

In September 1976, also the covariant world-sheet formulation of the spinning string was presented in two papers [38] by Brink, Di Vecchia and Howe and by Deser and Zumino. In retrospect this can be considered as a crucial ingredient for the Polyakov formulation [39] of spinning strings with arbitrary world-sheet topology. In this respect, $(p+1)$ supergravity is necessary for the consistent formulation of any $p$-dimensional extended object coupled to fermions. 
In the subsequent years all higher extended $4 D$ supergravities with $N=5,6$ and 8 were constructed.

The maximally extended supergravity $(N=8)$ was found by Cremmer and Julia 40], by dimensional reduction of $11 D$ supergravity previously obtained by the same authors with Scherk (1978 [41), and its gauged version, accompanied with an $\mathrm{SO}(8)$ Yang-Mills symmetry, by de Wit and Nicolai (1982 [42]). Gell-Mann had earlier observed that SO(8) cannot accommodate the observable gauge symmetry $\mathrm{SU}(3) \times \mathrm{SU}(2) \times \mathrm{U}(1)$ of electroweak and strong interactions. However, it was later observed by Ellis, Gaillard, Maiani and Zumino (1982 443) that a hidden local SU(8) symmetry (found by Cremmer and Julia) could be identified as a viable Grand Unified Theory (GUT) for non-gravitational interactions. The basic assumption was that the degrees of freedom of the $\mathrm{SU}(8)$ gauge bosons could be generated at the quantum level, as it was known to occur in certain $2 D \sigma$-models, following the analysis of Di Vecchia, D'Adda and Lüscher 44]. However, contrary to $2 D \sigma$-models, which are renormalizable and therefore consistent quantum field theories, it turned out later that $N=8$ supergravity in $D=4$, which is also a kind of generalized $\sigma$-model, is unlikely to enjoy a similar property. This is one of the reasons why this attempt was abandoned. Another reason was closely related to the forthcoming string revolution, when Green and Schwarz (GS) (1984 [45]) proved that $D=10$, $N=1$ supergravity, coupled to supersymmetric Yang-Mills matter, could be embedded in a consistent superstring theory for a particular choice of gauge groups $\left(\mathrm{SO}(32)\right.$ and $\left.\mathrm{E}_{8} \times \mathrm{E}_{8}\right)$.

The GOS and GS papers gave strong evidence that superstrings consistent with space-time supersymmetry containing supergravity + matter (rather than pure higher extended supergravity), in the massless sector, were a possible candidate for a theory of quantum gravity, encompassing the other gauge interactions and free from unphysical ultraviolet divergences. On the contrary, in the context of point-field theories, these systems, even if the symmetries dictated in an almost unique way all the couplings, were found to be non-renormalizable, already at one loop, when standard perturbative techniques were applied to them (Grisaru, van Nieuwenhuizen, Vermaseren, 1976 [46]). Indeed it was later shown that this was also the case for pure supergravities at and beyond three loops. [These theories had, however, the remarkable property of being one- and two-loop finite (Grisaru, van Nieuwenhuizen, Vermaseren [46]; Grisaru [47]; Tomboulis [47]).] Pioneering work, in the late 70's, was also the analysis of spontaneous supersymmetry breaking in global and local supersymmetry. In rigid supersymmetry, Fayet 48] opened the way to the construction of the minimal supersymmetric extension of the Standard Model (MSSM), which in particular demanded two Higgs doublets. However, the gauge and supersymmetry breaking introduced by him required more degrees of freedom than the MSSM.

When supersymmetry is gauged, i.e. in supergravity, the supersymmetric version of the Higgs mechanism appears (super-Higgs), i.e. the goldstino is eaten up by the spin-3/2 gravitino (the gauge fermion of supergravity, the partner of the gravitons), which then becomes massive.

The possibility of having spontaneously broken supergravity with vanishing cosmological constant was shown by Deser and Zumino (Apr. 1977 [49]) and proved in detail by Cremmer 
et al. (Aug. 1978 [50]), by studying the most general matter coupling to $N=1$ supergravity for a chiral multiplet, whose superpotential triggers a non-vanishing gravitino mass. The Higgs effect for Goldstone fermions had also been considered earlier by Volkov and Soroka [51].

Another important result at that time, found by Zumino (Aug. 1979 [52]), was the fact that the most general supergravity couplings of chiral multiplets (with two-derivative action) were described by Kählerian $\sigma$-models.

Again, in retrospect, this Kählerian structure and the generalization thereof have played a role in superstring theory from both the world-sheet and target-space points of view.

Although in the 70's the work done in supersymmetric models for particle physics (using renormalizable Lagrangians with spontaneously broken supersymmetry) and that towards a deeper understanding of the structure of supergravity theories (off-shell formulations, matter couplings, etc.) went in parallel, with small intersections, they came closer and became eventually deeply connected after two major developments were made in the early 80's.

The first was the call made upon supersymmetry breaking near the electroweak scale, to solve the so-called hierarchy problem of gauge theories with fundamental Higgs scalars (Gildener, Weinberg; Veltman; Witten; Maiani) [53], [54].

This development and general properties of criteria for supersymmetry breaking, contained in two pivotal papers by Witten (Apr. 1981, Jan. 1982 [54]), opened up the field of supersymmetry and supergravity as main stream research in the United States and in the rest of the world.

The hierarchy problem is connected to the unnaturalness of the hierarchy $E_{\mathrm{F}} / E_{X}\left(E_{\mathrm{F}}\right.$ being the Fermi scale) in any renormalizable theory with fundamental scalars, whose v.e.v. triggers the electroweak gauge symmetry breaking at a scale $E_{\mathrm{F}}$ much lower than any other (cut-off) scale $E_{X}$.

This is due to the quadratic dependence on the cut-off $\Lambda$ of the effective potential, which, at one loop, manifests itself in a term

$$
\sum_{J_{i}}(-)^{2 J_{i}}\left(2 J_{i}+1\right) \mathcal{M}_{J_{i}}^{2}(\phi) \Lambda^{2}
$$

where $M_{J_{i}}^{2}(\phi)$ are the (scalar) field-dependent masses of particle species with spin $J_{i}$. In an arbitrary supersymmetric renormalizable field theory with no traceful Abelian gauge group factor, the expression multiplying $\Lambda^{2}$ identically vanishes (owing to the special relation between boson and fermion couplings), as was shown by Girardello, Palumbo and the author (Apr. 1979 [55]). This is also true for matter-coupled $N=1$ supergravity with a single chiral multiplet on a flat Kähler manifold (1978 [50]) and in spontaneously broken extended supergravity via the Scherk-Schwarz mechanism (1979 [56]).

However, a closer look at boson-fermion mass matrices revealed that this property made models previously considered by Fayet more problematic, since they tended either to give an unrealistic spectrum with some scalar superpartners of quarks and leptons lighter than their 
fermionic counterparts, or to need a traceful additional U(1) gauge interaction, plagued with triangular anomalies. Cancelling these anomalies usually needed extra fields, which eventually allowed vacua with broken colour or charge symmetry.

However, when the most general coupling of $N=1$ supergravity to an arbitrary matter system, with arbitrary gauge interactions, became available (Cremmer, Ferrara, Girardello, Van Proeyen, 1982 [57]), it was realized that, provided $m_{3 / 2} \ll M_{\mathrm{Pl}}$ and possibly $\simeq O(\mathrm{TeV})$, mass terms for any observable scalar $O\left(m_{3 / 2}\right)$ were easily generated, thus resolving the partnerspartner splitting problem, which generally occurred in spontaneously broken rigid theories.

There is an alternative way of phrasing this: in the Fayet-type models, the goldstino has coupling to the observable sector $O(1)$ and the gravitino mass is very tiny, $m_{3 / 2} \sim 10^{-13} \mathrm{GeV}$, while in supergravity models with $m_{3 / 2} \gtrsim O\left(m_{\mathrm{Z}}\right)$ the goldstino coupling is highly suppressed $\left[O\left(m_{3 / 2} / M_{\mathrm{Pl}}\right)\right]$, which implies that the gravitino only carries gravitational interactions (Fayet [58]).

In the limit in which $m_{3 / 2}$ is kept fixed and couplings $O\left(1 / M_{\mathrm{Pl}}\right)$ are neglected, spontaneously broken supergravity models behave as globally supersymmetric models with softly broken terms, i.e. terms with dimension $\leq 3$, which do not induce quadratic divergences in the low-energy effective theory.

These terms had been classified in 1981 by Girardello and Grisaru [59]. A generalization of non-renormalization theorems for superpotential terms in a generic theory were also found using superspace techniques, by Grisaru, Siegel, Roček (June 1979 [60]).

Softly broken terms and renormalization theorems were used to construct viable supersymmetric GUTs, including the MSSM as their low-energy effective theory, with no hierarchy problem (the first of these was constructed by Georgi and Dimopoulos in the summer of 1981 [61]). Soon after, realistic electroweak and GUT models, with spontaneously broken supersymmetry triggered by the supergravity couplings at the tree level, were constructed (Barbieri, Ferrara, Savoy, 1982 [62]; Chamseddine, Nath, Arnowitt, 1982 [63]; Hall, Lykken, Weinberg 1983 [64]). A general feature of these models is that the messengers of supersymmetry breaking to the observable sector (encompassing electroweak and strong interactions) are a set of neutral chiral multiplets (called the hidden sector), which have only gravitational interactions and decouple from the low-energy theory; in the latter, the only trace of them is to produce the soft-breaking terms, then having the effect of modifying the supertrace formula of global sypersymmetry with an additional (field-independent) constant (with no physical consequences on the theory decoupled from gravity).

Nowadays, in the MSSM, the electroweak symmetry is broken through radiative corrections, through a Coleman-Weinberg mechanism, while supersymmetry is broken at the tree level through the soft-breaking terms.

Considering the initial condition for the couplings as given at the Planck scale and evolving them through the renormalization group equations (Ibàñez, Ross in 1981 [65], Alvarez-Gaumé, Polchinski, Wise in 1982 [66]), in a region of the parameter space, the electroweak symmetry is indeed found to be spontaneously broken with a Higgs mass of the same order of magnitude as 
the gravitino mass. There is a particular subclass of spontaneoulsy broken supergravity models, called no-scale supergravities (Cremmer, Ferrara, Kounnas and Nanopoulos, 1983 [67]; Ellis et al. 68), where the supergravity-breaking scale $m_{3 / 2}$ is arbitrary at the tree level (owing to flat directions in the superpotential). In these models, radiative corrections may generate the hierarchy $m_{3 / 2}=M_{\mathrm{Pl}} \mathrm{e}^{-c / g^{2}}$, then explaining how a small scale can be created in a theory whose only original dimensionful scale is $M_{\mathrm{Pl}}$.

It was later shown by Witten [69] that many $4 D$ superstring models have, in the point-field limit, a no-scale structure; therefore, after supersymmetry breaking, they may give rise to a dynamical hierarchy.

Nowadays almost every particle physicist knows what $\tan \beta, A, B$ represent in the general parametrization of the soft-breaking terms of the MSSM.

The second breakthrough was on physics at the Planck scale (Green, Schwarz, Sept. 1984 [45]), namely the discovery of anomaly-free $10 D$ supergravity coupled to Yang-Mills matter or consistent superstring theories, for specific gauge group choices (in open and heterotic strings) (Gross, Harvey, Martinec, Rohm, Nov. 1984 [70]). Heterotic string theories, after suitable compactification of six extra dimensions (on some compact manifolds with special properties), led to $N=1$ effective supergravity theories, with a spectrum of charged chiral multiplets (chiral with respect to the surviving gauge group $G^{\prime} \supset \mathrm{SU}(3) \times \mathrm{SU}(2) \times \mathrm{U}(1)$ (after compactification) and accommodating families of quarks and leptons with the $\mathrm{SU}(3) \times \mathrm{SU}(2) \times \mathrm{U}(1)$ assignment of the Standard Model.

The use of $10 D$ Yang-Mills fields, prior to compactification, is crucial to overcome previous difficulties encountered in Kaluza-Klein supergravities (Freund, Rubin, 1980 [71]; Witten, 1981 [72]; Duff, Nilsson, Pope, 1986 [73]), where attempts were made at obtaining the $\mathrm{SU}(3) \times \mathrm{SU}(2)$ $\times \mathrm{U}(1)$ gauge symmetries from the isometries of the internal manifold. In fact, even if in some cases the desired gauge group could be obtained (Witten, 1981 [72]; Castellani, D'Auria, Fré, 1984 [74]), these failed because the resulting fermion spectrum was not chiral with respect to the electroweak gauge symmetry.

In models where supersymmetry breaking occurs via a non-trivial dilaton superpotential, the neutral fields coming from the internal components of the metric tensor $G_{I J}$ are natural candidates for flat directions, at least in the limit of manifolds that are "large" with respect to the string scale.

In particular, in $4 D$ heterotic superstring theories, compactified on Calabi-Yau manifolds (Candelas, Horowitz, Strominger, Witten, Jan. 1985 [75]), or their "orbifold limit" [76], a natural identification of the hidden sector occurs with a set of "moduli fields", which parametrize the deformations of the Kähler class and complex structure of the manifold (generalization of radial deformations of simple tori) [77]. A popular scenario for a non-perturbative dilaton superpotential is the gaugino-condensation mechanism (Ferrara, Girardello, Nilles [78]) in the hidden sector gauge group (Derendinger, Ibáñez, Nilles; Dine, Rohm, Seiberg, Witten [79]). The fact that some moduli remain large (with respect to the string scale) could result in a sliding gravitino mass, which could then be stabilized through radiative corrections in the observable 
sector with a dynamical suppression with respect to $M_{\mathrm{Pl}}$.

In recent years a suggestion has been made (Duff [80]; Strominger [81]) that strings may, in the strong coupling regime, have a simpler formulation in terms of a dual theory (five-brane) in the weak coupling. These theories, in $D=10$, have the same field theory limit, namely, $D=10$ supergravity (which is unique because of supersymmetry), but electrically charged massive string states correspond to "magnetically" charged five-brane states (and vice versa) with a similar relation as it occurs between electric and magnetic charge in Dirac monopole quantization [82].

This is an explicit manifestation of the general fact that a $(p+1)$ form gauge field is, in $D$ dimensions, naturally coupled to a $p$-dimensional extended object, and that its "dual" potential (which is a $D-p-3$ form) is naturally coupled to a $D-p-4$ extended object. From topological arguments, similar to Dirac's, the product of the two couplings must be quantized.

In toroidal compactifications, it has indeed been shown (Sen and Schwarz [83]) that the spectrum of both electrically and magnetically charged states (the latter obtained from the low-energy effective field theory, i.e. an $N=4$ supergravity coupled to Yang-Mills) satisfies an $\mathrm{SL}(2, \mathrm{Z})$ duality for the dilaton chiral multiplet $S=\left(1 / g^{2}\right)+i \theta\left(g^{2}\right.$ is the field-dependent gauge coupling and $\theta$ is the field-dependent " $\theta$-angle"). This may therefore suggest a "modularinvariant" dilaton potential [84]. This symmetry is similar to the "moduli duality", which occurs in weakly coupled strings as a consequence of the world-sheet non-trivial topology 85.].

This approach is worth exploring, even if it is difficult to imagine that a microscopic, consistent quantum theory describing five-brane propagation could possibly exist.

Finally, let me conclude by making some remarks about the possible indirect experimental signals, indicating that supersymmetry may be just around the corner. With an optimistic attitude, these are

1) The non-observation of proton decay within the limit of a lifetime of $10^{32}$ years in the main channel $\mathrm{p} \rightarrow \pi^{0} \mathrm{e}^{+}$, excluding minimal GUT unification.

2) The LEP precision measurements, which are incompatible with gauge-coupling unification for conventional minimal GUTs, but are in reasonable agreement with minimal supersymmetric GUTs, with supersymmetry broken at the TeV scale.

3) The top Yukawa coupling, unusually large with respect to the one of other quarks and leptons.

4) The possible resolution of the dark-matter problem, with some of the neutral supersymmetric particles as natural dark-matter candidates.

Although none of these facts is per se a compelling reason for supersymmetry and may find alternative explanations, it is fair to say that they can all be explained in the context of a supersymmetric extension of ordinary gauge theories. 
Whatever the final theory (strings?) for quantum gravity will be, supergravity 866 remains a deep and non-trivial extension of the principle of general covariance and Yang-Mills gauge symmetry, which played such an important role in the description of natural phenomena.

Let us hope that nature has used this fascinating structure! 


\section{References}

[1] S. Ferrara and J.G. Taylor (eds.), Supergravity 81 (Cambridge Univ. Press, Cambridge, 1981);

S. Ferrara, J.G. Taylor and P. van Nieuwenhuizen (eds.), Supersymmetry and supergravity 82 (World Scientific, Singapore, 1982).

[2] Yu.A. Gol'fand and E.P. Likhtman, JETP Lett. 13 (1971) 323.

[3] D.V. Volkov and V.P. Akulov, Phys. Lett. 46B (1973) 109.

[4] B. Zumino, in Renormalization and invariance in quantum field theory, ed. E. Caianello (Plenum Press, New York, 1974), p. 367.

[5] J.-L. Gervais and B. Sakita, Nucl. Phys. B34 (1971) 633.

[6] A. Neveu and J. Schwarz, Nucl. Phys. B31 (1971) 86.

[7] P. Ramond, Phys. Rev. D3 (1971) 2415.

[8] J. Wess and B. Zumino, Nucl. Phys. B70 (1974) 39.

[9] A. Salam and J. Strathdee, Nucl. Phys. B76 (1974) 477.

[10] S. Ferrara, B. Zumino and J. Wess, Phys. Lett. 51B (1974) 239.

[11] J. Wess and B. Zumino, Phys. Lett. 49B (1974) 52.

[12] J. Iliopoulos and B. Zumino, Nucl. Phys. B76 (1974) 310.

[13] S. Ferrara, J. Iliopoulos and B. Zumino, Nucl. Phys. B77 (1974) 413.

[14] J. Wess and B. Zumino, Nucl. Phys. B78 (1974) 1.

[15] S. Ferrara and B. Zumino, Nucl. Phys. B79 (1974) 413.

[16] A. Salam and J. Strathdee, Phys. Lett. 51B (1974) 353.

[17] P. Fayet and J. Iliopoulos, Phys. Lett. 51B (1974) 461.

[18] S. Ferrara and B. Zumino, Nucl. Phys. B87 (1975) 207.

[19] J. Scherk and J. Schwarz, Nucl. Phys. B81 (1974) 118.

[20] P. Fayet, Phys. Lett. 58B (1975) 67.

[21] L. O'Raifeartaigh, Nucl. Phys. B6 (1975) 331.

[22] P. Fayet, Nucl. Phys. B113 (1976) 135. 
[23] M. Ademollo, L. Brink, A. D'Adda, R. D'Auria, E. Napolitano, S. Sciuto, E. del Giudice, P. Di Vecchia, S. Ferrara, F. Gliozzi, R. Musto and R. Pettorino, Phys. Lett. 62B (1976) 105.

[24] G. Gliozzi, J. Scherk and D. Olive, Phys. Lett. 65B (1976) 282; Nucl. Phys. B122 (1977) 253.

[25] M. Ademollo, L. Brink, A. D'Adda, R. D'Auria, E. Napolitano, S. Sciuto, E. Del Giudice, P. Di Vecchia, S. Ferrara, F. Gliozzi, R. Musto, R. Pettorino and J. Schwarz, Nucl. Phys. B111 (1976) 77.

[26] D. Gepner, Nucl. Phys. B296 (1988) 57; Phys. Lett. 199B (1987) 477;

W. Lerche, D. Lüst and A.N. Schellekens, Nucl. Phys. B287 (1987) 477;

H. Kawai, D.C. Lwellen and S.-H.H. Tye, Nucl. Phys. B298 (1987) 1;

L. Antoniadis, C.P. Bachas and C. Kounnas, Nucl. Phys. B289 (1987) 87.

[27] D.Z. Freedman, P. van Nieuwenhuizen and S. Ferrara, Phys. Rev. D13 (1976) 3214.

[28] S. Deser and B. Zumino, Phys. Lett. 62B (1976) 335.

[29] This nomenclature was inherited from the "dual models" that had started with the seminal paper by

G. Veneziano, Nuovo Cimento 57A (1968) 190.

[30] S. Ferrara, J. Scherk and P. van Nieuwenhuizen, Phys. Rev. Lett. 37 (1976) 1035.

[31] S. Ferrara, F. Gliozzi, J. Scherk and P. van Nieuwenhuizen, Nucl. Phys. B117 (1976) 333;

D.Z. Freedman and J.H. Schwarz, Phys. Rev. D15 (1977) 1007;

S. Ferrara, D.Z. Freedman, P. van Nieuwenhuizen, P. Breitenlohner, F. Gliozzi and J. Scherk, Phys. Rev. D15 (1973) 1013.

[32] S. Ferrara and P. van Nieuwenhuizen, Phys. Rev. Lett. 37 (1976) 1669.

[33] M.B. Green and J.H. Schwarz, Nucl. Phys. B181 (1981) 502; Phys. Lett. 109B (1982) 444 and 136B (1984) 367.

[34] E. Cremmer, J. Scherk and S. Ferrara, Phys. Lett. 68B (1977) 234.

[35] D.Z. Freedman, Phys. Rev. Lett. 38 (1977) 105;

S. Ferrara, J. Scherk and B. Zumino, Phys. Lett. 66B (1977) 35.

[36] S. Ferrara, J. Scherk and B. Zumino, Nucl. Phys. B121 (1977) 393.

[37] K. Narain, Phys. Lett. 169B (1986) 41;

K. Narain, M.H. Sarmadi and E. Witten, Nucl. Phys. B279 (1986) 96.

[38] L. Brink, P. Di Vecchia and P. Howe, Phys. Lett. 65B (1976) 471;

S. Deser and B. Zumino, Phys. Lett. 65B (1976) 369. 
[39] A.M. Polyakov, Phys. Lett. 103B (1981) 207 and 211.

[40] E. Cremmer and B. Julia, Phys. Lett. 80B (1978) 48; Nucl. Phys. B159 (1979) 141.

[41] E. Cremmer, B. Julia and J. Scherk, Phys. Lett. 76B (1978) 409.

[42] B. de Wit and H. Nicolai, Nucl. Phys. B208 (1982) 323.

[43] J. Ellis, M.K. Gaillard, L. Maiani and B. Zumino, in Unification of fundamental interactions, eds. S. Ferrara, J. Ellis and P. van Nieuwenhuizen [Plenum Press (Ettore Majorana Series), New York, 1980], p. 69;

J. Ellis, M.K. Gaillard and B. Zumino, Phys. Lett. 94B (1980) 343.

[44] A. D'Adda, P. Di Vecchia and M. Lüscher, Nucl. Phys. 146 (1978) 63 and B152 (1979) 125.

[45] M. Green and J. Schwarz, Phys. Lett. 149B (1984) 117.

[46] M.T. Grisaru, P. van Nieuwenhuizen and J.A.M. Vermaseren, Phys. Rev. Lett. 37 (1976) 1662 ;

P. van Nieuwenhuizen and J.A.M. Vermaseren, Phys. Lett. 65B (1976) 263.

[47] M.T. Grisaru, Phys. Lett. B66 (1977) 75;

E.T. Tomboulis, Phys. Lett. 67B (1977) 417.

[48] P. Fayet, Phys. Lett. 69B (1977) 489.

[49] S. Deser and B. Zumino, Phys. Rev. Lett. 38 (1977) 1433.

[50] E. Cremmer, B. Julia, J. Scherk, S. Ferrara, L. Girardello and P. van Nieuwenhuizen, Phys. Lett. 79B (1978) 231; Nucl. Phys. B147 (1979) 105.

[51] D.V. Volkov and V.A. Soroka, JETP Lett. 18 (1973) 312.

[52] B. Zumino, Phys. Lett. 87B (1979) 203.

[53] E. Gildener and S. Weinberg, Phys. Rev. D13 (1976) 3333;

S. Weinberg, Phys. Lett. 82B (1979) 387;

M. Veltman, Acta Phys. Polon. B12 (1981) 437;

L. Maiani, Proc. Summer School of Gif-sur-Yvette 1979 (IN2P3, Paris, 1979), p. 1.

[54] E. Witten, Nucl. Phys. B185 (1981) 513 and B202 (1982) 253.

[55] S. Ferrara, L. Girardello and F. Palumbo, Phys. Rev. D20 (1979) 403.

[56] J. Scherk and J. Schwarz, Nucl. Phys. B153 (1979) 61;

E. Cremmer, J. Scherk and J. Schwarz, Phys. Lett. 82B (1979) 60.

[57] E. Cremmer, S. Ferrara, L. Girardello and A. van Proeyen, Phys. Lett. 116B (1982) 231; Nucl. Phys. B212 (1983) 413. 
[58] P. Fayet, Phys. Lett. 70B (1977) 461.

[59] L. Girardello and M.T. Grisaru, Nucl. Phys. B194 (1982) 65.

[60] M.T. Grisaru, W. Siegel and M. Roček, Nucl. Phys. B159 (1979) 429.

[61] S. Dimopoulos and H. Georgi, Nucl. Phys. B193 (1981) 150.

[62] R. Barbieri, S. Ferrara and C. Savoy, Phys. Lett. 199B (1982) 343.

[63] A.H. Chamseddine, R. Arnowitt and P. Nath, Phys. Rev. Lett. 49 (1982) 970.

[64] L. Hall, J. Lykken and S. Weinberg, Phys. Rev. D27 (1983) 2359.

[65] L. Ibáñez and G.G. Ross, Phys. Lett. 105B (1981) 439 and 110B (1982) 215.

[66] L. Alvarez-Gaumé, J. Polchinski and M. Wise, Nucl. Phys. B221 (1983) 495.

[67] E. Cremmer, S. Ferrara, C. Kounnas and D.V. Nanopoulos, Phys. Lett. 133B (1983) 61.

[68] J. Ellis, A.B. Lahanas, D.V. Nanopoulos and K. Tamvakis, Phys. Lett. 134B *1984) 429;

J. Ellis, C. Kounnas and D.V. Nanopoulos, Nucl. Phys. B247 (1984) 373.

[69] E. Witten, Phys. Lett. 155B (1985) 151.

[70] D.J. Gross, J.A. Harvey, E. Martinec and R. Rohm, Phys. Rev. Lett. 54 (1985) 502.

[71] P.G.O. Freund and M.A. Rubin, Phys. Lett. 97B (1980) 233.

[72] E. Witten, Nucl. Phys. B186 (1981) 412.

[73] M.J. Duff, B.E.W. Nilsson and C.N. Pope, Phys. Rep. 130 (1986) 1.

[74] L. Castellani, R. D’Auria, and P. Fré, Nucl. Phys. B239 (1984) 60.

[75] P. Candelas, G. Horowitz, A. Strominger and E. Witten, Nucl. Phys. B258 (1985) 46.

[76] L. Dixon, J.A. Harvey, C. Vafa and E. Witten, Nucl. Phys. B261 (1985) 678.

[77] N. Seiberg, Nucl. Phys. B303 (1988) 286;

S. Cecotti, S. Ferrara and L. Girardello, Int. J. Mod. Phys. 4 (1989) 2475;

P. Candelas, P. Green and T. Hübsch, Nucl. Phys. B330 (1990) 49;

P. Candelas and X.C. de la Ossa, Nucl. Phys. B342 (1990) 246;

S. Ferrara and A. Strominger, in String '89, eds. R. Arnowitt, R. Brijan, M.J. Duff and D.V. Nanopoulos (World Scientific, Singapore, 1989), p. 245;

A. Strominger, Comm. Math. Phys. 133 (1990) 42;

L. Dixon, V.S. Kaplunovsky and J. Louis, Nucl. Phys. B329 (1990) 27;

S. Cecotti and C. Vafa, Nucl. Phys. B367 (1991) 359;

A. Ceresole, R. D'Auria, S. Ferrara, W. Lerche and J. Louis, Int. Mod. Phys. 8A (1993) 79 . 
[78] S. Ferrara, L. Girardello and H.P. Nilles, Phys. Lett. 125B (1983) 457.

[79] J.-P. Derendinger, L.E. Ibáñez and H.P. Nilles, Phys. Lett. 155B (1985) 65;

M. Dine, R. Rohm, N. Seiberg and E. Witten, Phys. Lett. 156B (1985) 55.

[80] M.J. Duff, Class. Quant. Grav. 5 (1988) 189;

M.J. Duff and J.X. Lu, Phys. Rev. Lett. 66 (1991) 1402.

[81] A. Strominger, Nucl. Phys. B343 (1990) 167;

C.G. Callan, J.A. Harvey and A. Strominger, Nucl. Phys. B359 (1991) 611.

[82] C. Montonen and D. Olive, Phys. Lett. B72 (1977) 117.

[83] A. Sen, Mod. Phys. Lett. A8 (1993) 2023;

J.H. Schwarz and A. Sen, Nucl. Phys. B411 (1994) 35; Phys. Lett. B312 (1993) 105.

[84] A. Font, L. Ibáñez, D. Lüst and F. Quevedo, Phys. Lett. B249 (1990) 35.

[85] K. Kikkawa and M. Yamasaki, Phys. Lett. 149B (1984) 357;

N. Sakai and L. Senda, Progr. Theor. Phys. 75 (1986) 692;

V.P. Nair, A. Shapere, A. Strominger and F. Wilczek, Nucl. Phys. B287 (1987) 402;

A. Giveon, E. Rabinovici and G. Veneziano, Nucl. Phys. B322 (1989) 167;

A. Shapere and F. Wilczek, Nucl. Phys. B320 (1989) 167;

M. Dine, P. Huet and N. Seiberg, Nucl. Phys. B322 (1989) 301;

J. Molera and B. Ovrut, Phys. Rev. D40 (1989) 1150;

J. Lauer, J. Mas and H.P. Nilles, Phys. Lett. B226 (1989) 251; Nucl. Phys. B351 (1991) 353 ;

W. Lerche, D. Lüst and N.P. Warner, Phys. Lett. B231 (1989) 417;

M. Duff, Nucl. Phys. B335 (1990) 610;

A. Giveon and M. Porrati, Phys. Lett. B246 (1990) 54; Nucl. Phys. B355 (1991) 442;

A. Giveon, N. Malkin and E. Rabinovici, Phys. Lett. B238 (1990) 57;

J. Erler, D. Jungnickel and H.P. Nilles, Phys. Lett. B276 (1992) 303;

S. Ferrara, D. Lüst, A. Shapere and S. Theisen, Phys. Lett. B233 (1989) 147;

L. Dixon, V. Kaplunovsky and J. Louis, Nucl. Phys. B355 (1991) 649;

G.L. Cardoso and B. Ovrut, Nucl. Phys. B369 (1992) 351 and B392 (1993) 315;

J.-P. Derendinger, S. Ferrara, C. Kounnas and F. Zwirner, Nucl. Phys. B372 (1992) 145;

L. Antoniadis, K. Narain and T. Taylor, Phys. Lett. B267 (1991) 37;

L. Antoniadis, E. Gavai and K. Narain, Nucl. Phys. B365 (1992) 93.

[86] Reviews on the developments of supergravity in the 80's can be found in:

P. van Nienwenhuizen, Phys. Rep. 68 (1981) 189;

H.P. Nilles, Phys. Rep. 110 (1984) 1;

S. Ferrara, vol. 2 of Supersymmetry (North Holland, Amsterdam/World Scientific, Singapore, 1987). 